\title{
Effect of cytomegalovirus infection on breastfeeding transmission of HIV and on the health of infants born to HIV- infected mothers
}

\author{
Tiffany S. Chang ${ }^{\mathrm{a}}$, Jeffrey Wiener ${ }^{\mathrm{b}}$, Sheila C. Dollard ${ }^{\mathrm{b}}$, Minal M. Amin ${ }^{\mathrm{b}}$, Sascha Ellington ${ }^{\mathrm{b}}$, \\ Charles Chasela ${ }^{c}$, Dumbani Kayirac, Gerald Tegha ${ }^{c}$, Deborah Kamwendoc, Denise J. \\ Jamieson $^{b}$, Charlie van der Horst ${ }^{d}$, and Athena P. Kourtis ${ }^{b}$ for the BAN Study Team \\ aEmory University School of Medicine, Atlanta, Georgia, USA \\ bUS Centers for Disease Control and Prevention, Atlanta, Georgia, USA \\ 'UNC Project, Lilongwe, Malawi \\ dUniversity of North Carolina (UNC) at Chapel Hill, Chapel Hill, North Carolina, USA
}

\begin{abstract}
Background-Cytomegalovirus (CMV) infection can be acquired in utero or postnatally through horizontal transmission and breastfeeding. The effect of postnatal CMV infection on postnatal HIV transmission is unknown.
\end{abstract}

Methods-The Breastfeeding, Antiretrovirals and Nutrition study, conducted in Malawi, randomized 2369 mothers and their infants to three antiretroviral prophylaxis arms -mother (triple regimen), infant (nevirapine), or neither - for 28 weeks of breastfeeding, followed by weaning. Stored plasma and peripheral blood mononuclear cell specimens were available for 492 infants at 24 weeks and were tested with CMV PCR. Available samples from infants who were CMV PCRpositive at 24 weeks were also tested at birth $(N=242)$, and from infants PCR-negative at 24 weeks were tested at 48 weeks $(N=96)$. Cox proportional-hazards models were used to determine if CMV infection was associated with infant morbidity, mortality, or postnatal HIV acquisition.

Results-At 24 weeks of age, CMV DNA was detected in 345/492 infants (70.1\%); the estimated congenital CMV infection rate was $2.3 \%$, and the estimated rate of CMV infection at 48 weeks was $78.5 \%$. CMV infection at 24 weeks was associated with subsequent HIV acquisition through breastfeeding or infant death between 24 and 48 weeks of age (hazard ratio 4.27, $P=$ $0.05)$.

Conclusion-Most breastfed infants of HIV-infected mothers in this resource-limited setting are infected with CMV by 24 weeks of age. Early CMV infection may be a risk factor for subsequent

Correspondence to: Athena P. Kourtis, MD, PhD, MPH, Division of Reproductive Health, NCCDPHP, Centers for Disease Control and Prevention, Professor of Pediatrics, Emory University School of Medicine, 4770 Buford Highway, NE, MSF74, Atlanta, GA 30341, USA. Tel: +1 770488 5216; fax: +1 770488 6391; apk3@cdc.gov.

Disclaimer: The findings and conclusions in this article are those of the authors and do not necessarily represent the official position of the Centers for Disease Control and Prevention.

Conflicts of interest

There are no conflicts of interest. 
infant HIV infection through breastfeeding, pointing to the need for comprehensive approaches in order to achieve elimination of breastfeeding transmission of HIV.

\section{Keywords}

breastfeeding; cytomegalovirus; HIV; infant; mother-to-child transmission

\section{Introduction}

Congenital cytomegalovirus (CMV) infection - a result of intrauterine mother-to-infant transmission - is the most common congenital infection [1,2]. Postnatal acquisition of CMV can occur through horizontal transmission and through breastfeeding [3], and is thought to be generally benign in healthy full-term infants. However, premature infants or other groups of immuno-compromised infants are at risk for more extensive disease [4,5], and may also experience long-term cognitive delays [6].

Sub-Saharan Africa remains the region of the world most heavily affected by HIV-1; more than $90 \%$ of all children who acquired HIV-1 in 2011 resided in the sub-Saharan Africa. The interaction of CMV infection and HIV-1 transmission to the infant is complex and poorly understood. Studies have suggested that mother-to-child HIV transmission is more frequent among newborns with congenital CMV infection, and that infants with both infections experience higher rates of HIV disease progression, neurodevelopmental deficits, and death [7-11]. The effect of CMV infection on infants who are HIV-1-exposed but not infected has also received recent attention. In areas with high HIV-1 prevalence, there is some evidence that HIV-1-exposed but uninfected infants may be a population at risk for increased morbidity and mortality [12,13], decreased growth and psychomotor development [6], and immunologic abnormalities [14]. Whether postnatal CMV infection of the infant correlates with transmission of HIV-1 infection through breastfeeding has not, to our knowledge, been studied. Furthermore, whether maternal receipt of HAART may affect CMV transmission to the infant is unclear. The introduction of HAART in HIV-1-infected pregnant women has been associated with decreased rates of congenital and early postnatal CMV infection $[15,16]$. The role of postnatal HAART in reducing CMV transmission has not been evaluated to date.

The objectives of this study were: to delineate the relationship of congenital and postnatal CMV and HIV transmission in a cohort of infants born to HIV-1-infected mothers; to characterize any effects of CMV infection on the health and growth of these infants; and to determine whether HAART administered to the mothers during breastfeeding affected postnatal CMV transmission to the infants. We used samples collected and stored from infants enrolled in the Breastfeeding, Antiretrovirals and Nutrition (BAN) study [17], who breastfed for 28 weeks and were followed for their first 48 weeks of life in Lilongwe, Malawi. 


\section{Methods}

\section{Study population and testing strategy}

The BAN study was a randomized, controlled clinical trial that evaluated, in a factorial design, the safety and efficacy of: antiretroviral prophylaxis (maternal triple-drug antiretroviral regimen vs. infant daily nevirapine administered during 28 weeks of breastfeeding vs. a control arm of only one week of antiretroviral prophylaxis after delivery); and a maternal nutritional supplement during breastfeeding, in reducing postnatal mother-to-child HIV-1 transmission and in enhancing maternal health during breastfeeding [17]. The study randomized and followed 2369 mother-infant pairs between 2004 and 2010 in Lilongwe, Malawi; enrolled infants had to have a birth weight of at least $2000 \mathrm{~g}$ and be HIV-uninfected by 2 weeks of age.

Information on the cumulative risk of HIV transmission, infant death, and serious morbidity outcomes, as well as anthropometric data, was available for all study infants. We used stored infant plasma and peripheral blood mononuclear cell (PBMC) specimens from birth, 24 weeks, and 48 weeks of age, in order to detect congenital, early postnatal, and late postnatal CMV infection, respectively. Both stored plasma and PBMC specimens were available for 492 infants at 24 weeks and were tested with CMV PCR. Available samples from infants who were positive by PCR at 24 weeks were also tested at birth $(N=242)$ to determine the congenital CMV infection rate. Available samples from those infants who were CMV PCRnegative at 24 weeks were tested at 48 weeks $(N=96)$. An additional set of specimens from 30 of the 119 infants who were HIV PCR-positive by 2 weeks of age (and excluded from further follow-up in the BAN study) was also tested, to derive the congenital CMV infection rate among infants HIV-infected in utero/intrapartum. This study was approved by the Institutional Review Boards of the University of North Carolina at Chapel Hill and by the Centers for Disease Control and Prevention.

\section{Cytomegalovirus PCR}

Total DNA was purified from plasma and PBMC specimens using Qiagen blood mini kits on the Qiacube automated DNA extractor (Qiagen, Valencia, California, USA). CMV load was measured by Taqman-based PCR using Perfecta Fastmix (Quanta Biosciences Inc., Gaithersburg, Maryland, USA) with amplification using the MX 3005P Real-time PCR System (Agilent Technologies, New Castle, Delaware, USA). PCR targeted the highly conserved viral immediate early 2 gene as described by Boppana et al. [18].

\section{Statistical analysis}

The rate of CMV infection as measured by CMV PCR and median CMV viral load was calculated at birth, 24 weeks, and 48 weeks, and separately by antiretroviral treatment group and infant HIV infection. To estimate the CMV infection rate at birth, all infants with a negative CMV PCR result at 24 weeks were assumed to also be negative at birth. To estimate the CMV infection rate at 48 weeks, all infants with a positive CMV PCR result at 24 weeks were assumed to also be positive at 48 weeks. The estimated infection rates at birth and 48 weeks were adjusted for missing data by multiplying the number with assumed results by the percentage of those with available specimens. 
Rates of CMV infection were compared between groups using chi-square or Fisher's exact test, and viral loads were compared using Wilcoxon rank-sum tests. Relative risks were calculated to examine the effect of potential predictors of CMV infection at 24 weeks, including maternal age, $\mathrm{CD} 4^{+} \mathrm{T}$-cell count, maternal HIV load, infant birth weight, and infant white blood cell count at birth. To examine the effect of CMV infection on negative health outcomes in HIV-exposed infants, we included CMV PCR at 24 weeks as a covariate in mixed-effects models for infant growth and in Cox proportional-hazards regression models for serious infant morbidity requiring hospitalization (including severe pneumonia or serious febrile illness, severe diarrhea or growth faltering, and malaria), mortality, and HIV acquisition through breastfeeding (after 2 weeks of age). Results were considered statistically significant when $P$ was less than 0.05 .

\section{Results}

Only 492 of the 2369 infants enrolled in the BAN study had available stored plasma and PBMC specimens at 24 weeks of age and were tested for CMV. To ensure this subset was representative of the entire BAN study, demographic variables, maternal $\mathrm{CD} 4^{+}$cell count, infant characteristics at delivery, and infant HIV infection through breastfeeding were compared between the subset and all other infants without available specimens. No significant differences were found.

\section{Detection of cytomegalovirus DNA in the infants}

At 24 weeks of age, CMV DNA (either in plasma or in PBMC) was detected in 345 of 492 infants [70.1\%; 95\% confidence interval (CI) 65.9\%, 74.1\%]. The median CMV plasma viral load at 24 weeks of age was 176.4 copies/ml [interquartile range (IQR) 88.6-433.3]. Of the CMV PCR-positive infants at 24 weeks and with available specimens at birth $(n=242)$, eight were also CMV PCR-positive at birth, with an estimated congenital CMV infection rate (as detected by positive plasma or PBMC CMV PCR) of 2.3\% (95\% CI 1.0\%, 4.5\%) (adjusted for missing data). All eight cases of congenital CMV infection were among infants who remained HIV-uninfected until their last study visit (six of eight completed 48 weeks). Of those CMV PCR-negative at 24 weeks with available specimens at 48 weeks, 27 of 96 (28.1\%) were CMV PCR-positive at 48 weeks of age, for an estimated cumulative rate of CMV infection (as detected by plasma/PBMC PCR) of 78.5\% (95\% CI 73.6\%, 82.9\%) at 48 weeks (adjusted for missing data). The median CMV plasma viral load was $197.8 \mathrm{copies} / \mathrm{ml}$ (IQR 89.3-843.2) at birth and 61.3 copies/ml (IQR 30.7-111.7) at 48 weeks. The median CMV plasma viral load at 48 weeks was significantly lower than the respective values at birth $(P=0.02)$ and 24 weeks $(P<0.001)$.

An additional 30 birth specimens from infants HIV-PCR-positive at birth $(n=29)$ or at 1 week of age $(n=1)$ (who were not further followed in the BAN study and thus not included in the above group) were tested by CMV PCR at birth. We found that three $(10.0 \%$; $95 \%$ CI $2.1 \%, 26.5 \%$ ) of these specimens were also positive by CMV PCR, which is significantly higher than the estimated $2.3 \%$ congenital CMV infection rate in the HIV-exposed uninfected infants $(P=0.049)$. 


\section{Predictors of cytomegalovirus DNA detection and cytomegalovirus viral load levels at 24 weeks}

Infants with detectable CMV DNA at 24 weeks had mothers with lower antenatal CD4 ${ }^{+} \mathrm{T}$ cell count [relative risk $(\mathrm{RR})=0.95$ for each $100 \mathrm{cell} / \mu \mathrm{l}$ increase; $P=0.002$ ], higher HIV viral load during pregnancy $\left(\mathrm{RR}=1.27\right.$ for each 1 unit increase in $\log _{10}$ viral load; $P<$ $0.0001)$, and had lower birth weight ( $\mathrm{RR}=0.99$ for each $100 \mathrm{~g}$ increase; $P=0.047)$. The prevalence of infants with detectable CMV DNA at 24 weeks was similar for each study arm (70.2, 68.5, and $73.3 \%$ for the maternal antiretroviral, infant antiretroviral, and no-extended antiretroviral arm, respectively).

Cytomegalovirus plasma viral load was significantly higher in infants postnatally HIVinfected by 24 weeks, compared to infants HIV-uninfected at 24 weeks (median 1258.6 vs. 174.2 copies $/ \mathrm{ml} ; P=0.045$ ). For infants HIV-uninfected at 24 weeks, CMV viral load was higher in those who became HIV-infected by 48 weeks compared to those who remained HIV-uninfected at 48 weeks, but not significantly so (median 380.1 vs. 172.0 copies $/ \mathrm{ml} ; P=$ 0.095).

\section{Association of cytomegalovirus DNA detection at 24 weeks with HIV acquisition through breastfeeding and with infant morbidity, mortality, and growth}

Among nine infants who became HIV-infected between 24 and 48 weeks of age, the prevalence of CMV DNAemia at 24 weeks of age was $88.9 \%$, as compared with a prevalence of $69.8 \%$ among 476 who remained HIV-uninfected $(P=0.29)$, and a prevalence of $66.7 \%$ among six infants who became HIV-infected between 2 and 24 weeks of age $(P=$ 0.53). CMV DNAemia at 24 weeks showed a nonsignificant association with subsequent acquisition of HIV infection between 24 and 48 weeks (hazard ratio 4.52, $P=0.15$ ), with infant mortality by 48 weeks of age (hazard ratio $4.05, P=0.18$ ) and with the combined measure of HIV infection or infant death between 2 and 24 weeks of age (hazard ratio 2.49, $P=0.09$ ) (Table 1). A significant association was observed when the outcomes of HIV infection and infant death were combined between 24 and 48 weeks of age (hazard ratio $4.27, P=0.05)$. Kaplan-Meier estimates of the cumulative risk of HIV infection or death between 24 and 48 weeks of age by CMV DNAemia at 24 weeks are depicted in Fig. 1 (logrank $P=0.03$ ).

Cytomegalovirus DNAemia was not associated with severe morbidity outcomes requiring hospitalization, or with decreased infant weight-for-age or length-for-age (Table 1).

\section{Discussion}

To our knowledge, this is the first study to date to specifically examine the relationship between CMV infection in HIV-exposed infants and postnatal transmission of HIV through breastfeeding, and to investigate the relationship between postnatal antiretroviral treatment and postnatal CMV acquisition. Our study shows that the majority of infants born to HIVinfected mothers in a resource-limited setting practicing breastfeeding acquire CMV infection rapidly in their first year of life ( $70.1 \%$ by 24 weeks, $78.5 \%$ by 48 weeks). The estimated congenital CMV infection rate in infants HIV-uninfected at birth (as determined 
by DNA PCR of plasma/PBMC) was $2.3 \%$; in contrast, infants who were HIV-infected at birth had a congenital CMV infection rate of $10 \%$.

Past studies have shown similar high rates of CMV infection in HIV-exposed uninfected infants living in resource-limited settings [6,19], and have also suggested that the rate of congenital CMV infection may also be higher in these populations. A recent study found a congenital CMV rate of $3.8 \%$ in neonates admitted to a large referral center in sub-Saharan Africa (by CMV PCR of serum, urine, and saliva), with a rate of $11.4 \%$ in those born to HIV-infected mothers [20]. Our findings confirm previous findings of a very high rate of congenital CMV infection in HIV-infected infants [7,10,15,19,21].

In previous studies, infant CMV infection among HIV-uninfected neonates was associated with maternal age, antiretroviral treatment, $\mathrm{CD} 4^{+}$cell count, plasma, and breast milk viral load $[15,22]$. In the current study, CD4 ${ }^{+}$cell count and plasma HIV viral load were found to be significantly associated with infant CMV infection, whereas maternal age and antiretroviral status were not. The BAN study, however, did not initiate antiretrovirals until the time of labor. High levels of CMV in breast milk of HIV-infected mothers were demonstrated throughout lactation, with highest levels in the first 24 weeks postpartum [22], which correlates with the time of postnatal infant CMV acquisition we observed.

We found no significant correlation between CMV DNAemia and severe infant morbidity or decreased growth. These findings are different from those of a previous study among HIVexposed infants [6], and may be due to the low levels of CMV observed in our study infants. However, our findings show that detection of CMV DNA in the blood at 24 weeks was associated with the combined outcome of HIVacquisition or death from 24 to 48 weeks of age, which is a novel finding. The mechanisms underlying this association may be through CMV-mediated enhancement of HIV shedding in breast milk [23]. CMV in breast milk could be a co-factor that influences level of mucosal shedding of HIV, even in women receiving HAART.

To our knowledge, our study is the first to examine the effect of maternal antiretrovirals on the risk of postnatal transmission of CMV. The lack of an effect suggests that initiation of antiretroviral therapy prenatally may be necessary in order to allow time for CMV levels in breast milk (or other secretions) to decrease. Alternatively, the lack of an association with antiretroviral therapy may be due to an overall high level of CMV exposure in this population, possibly not related to breastfeeding.

One limitation to our study was the use of only blood specimens for PCR detection of congenital CMV, as urine or saliva was not available. It is thus possible that the congenital CMV DNAemia rates we report are underestimates of the true congenital CMV infection rates. Another limitation of the study was the small number of BAN infants with available specimens (492/2369), which may not have been fully representative of the entire study population.

These limitations notwithstanding, our study has many strengths. First, we had access to specimens from different time points in the infant's first year of life, allowing us to more closely determine timing of CMV acquisition. In addition, because of length of follow-up 
for up to 48 weeks, we were able to incorporate data on morbidity and mortality for up to a year of an infant's life.

In conclusion, our data indicate that early postnatal CMV infection in an HIV-exposed infant may predict subsequent HIV transmission through breastfeeding and infant mortality, suggesting that preventing or delaying CMV infection may offer advantages for HIV prevention. The role of maternal antiretroviral treatment, or of other possible adjunct approaches targeting $\mathrm{CMV}$, in decreasing the burden of this important co-infection, need to be further investigated.

\section{Acknowledgments}

We are grateful to the following: BAN Study Team at University of North Carolina Chapel Hill, Centers for Disease Control and Prevention, Atlanta, and UNC Project team in Lilongwe including: Linda Adair, Yusuf Ahmed, Mounir Ait-Khaled, Sandra Albrecht, Shrikant Bangdiwala, Ronald Bayer, Margaret Bentley, Brian Bramson, Emily Bobrow, Nicola Boyle, Sal Butera, Charles Chasela, Charity Chavula, Joseph Chimeran-g'ambe, Maggie Chigwenembe, Maria Chikasema, Norah Chikhungu, David Chilongozi, Grace Chiudzu, Lenesi Chome, Anne Cole, Amanda Corbett, Amy Corneli, Anna Dow, Ann Duerr, Henry Eliya, Sascha Ellington, Joseph Eron, Sherry Farr, Yvonne Owens Ferguson, Susan Fiscus, Valerie Flax, Ali Fokar, Shannon Galvin, Laura Guay, Chad Heilig, Irving Hoffman, Elizabeth Hooten, Mina Hosseinipour, Michael Hudgens, Stacy Hurst, Lisa Hyde, Denise Jamieson, George Joaki (deceased), David Jones, Elizabeth Jordan-Bell, Zebrone Kacheche, Esmie Kamanga, Gift Kamanga, Coxcilly Kampani, Portia Kamthunzi, Deborah Kamwendo, Cecilia Kanyama, Angela Kashuba, Damson Kathyola, Dumbani Kayira, Peter Kazembe, Caroline C. King, Rodney Knight, Athena P. Kourtis, Robert Krysiak, Jacob Kumwenda, Hana Lee, Edde Loeliger, Dustin Long, Misheck Luhanga, Victor Madhlopa, Maganizo Majawa, Alice Maida, Cheryl Marcus, Francis Martinson, Navdeep Thoofer, Chrissie Matiki (deceased), Douglas Mayers, Isabel Mayuni, Marita McDonough, Joyce Meme, Ceppie Merry, Khama Mita, Chimwemwe Mkomawanthu, Gertrude Mndala, Ibrahim Mndala, Agnes Moses, Albans Msika, Wezi Msungama, Beatrice Mtimuni, Jane Muita, Noel Mumba, Bonface Musis, Charles Mwansambo, Gerald Mwapasa, Jacqueline Nkhoma, Megan Parker, Richard Pendame, Ellen Piwoz, Byron Raines, Zane Ramdas, John Rublein, Mairin Ryan, Ian Sanne, Christopher Sellers, Diane Shugars, Dorothy Sichali, Wendy Snowden, Alice Soko, Allison Spensley, Jean-Marc Steens, Gerald Tegha, Martin Tembo, Roshan Thomas, Hsiao-Chuan Tien, Beth Tohill, Charles van der Horst, Esther Waalberg, Elizabeth Widen, Jeffrey Wiener, Cathy Wilfert, Patricia Wiyo, Innocent Zgambo, Chifundo Zimba. Finally and most especially, all the women and infants that have agreed to participate in the study.

Funding: The Breastfeeding, Antiretrovirals, and Nutrition Study was supported by grants from the Prevention Research Centers Special Interest Project of the Centers for Disease Control and Prevention (SIP 13-01 U48CCU409660-09, SIP 26-04 U48-DP000059-01, and SIP 22-09 U48-DP001944-01); the National Institute of Allergy and Infectious Diseases, the University of North Carolina Center for AIDS Research (P30-AI50410); the NIH Fogarty AIDS International Training and Research Program (DHHS/NIH/FIC 2-D43 Tw01039-06 and R24 Tw00798; the American Recovery and Reinvestment Act); and the Bill and Melinda Gates Foundation (Grant \# OPP53107). The antiretrovirals used in the BAN study were donated by Abbott Laboratories, GlaxoSmithKline, Boehringer Ingelheim, Roche Pharmaceuticals, and Bristol-Myers Squibb. The Call to Action PMTCT program was supported by the Elizabeth Glaser Pediatric AIDS Foundation, the United Nations Children's Fund, the World Food Program, the Malawi Ministry of Health and Population, Johnson \& Johnson, and the U.S. Agency for International Development.

\section{References}

1. Kenneson A, Cannon MJ. Review and meta-analysis of the epidemiology of congenital cytomegalovirus (CMV) infection. Rev Med Virol. 2007; 17:253-276. [PubMed: 17579921]

2. Dollard SC, Grosse SD, Ross DS. New estimates of the prevalence of neurological and sensory sequelae and mortality associated with congenital cytomegalovirus infection. Rev Med Virol. 2007; 17:355-363. [PubMed: 17542052]

3. Dworsky M, Yow M, Stagno S, Pass RF, Alford C. Cytomegalovirus infection of breast milk and transmission in infancy. Pediatrics. 1983; 72:295-299. [PubMed: 6310479]

4. Hamele M, Flanagan R, Loomis CA, Stevens T, Fairchok MP. Severe morbidity and mortality with breast milk associated cytomegalovirus infection. Pediatric Infect Dis J. 2010; 29:84-86. 
5. Lanzieri TM, Dollard SC, Josephson CD, Schmid DS, Bialek SR. Breast milk-acquired cytomegalovirus infection and disease in VLBW and premature infants. Pediatrics. 2013; 131:e1937-e1945. [PubMed: 23713111]

6. Gompels UA, Larke N, Sanz-Ramos M, Bates M, Musonda K, Manno D, et al. Human cytomegalovirus infant infection adversely affects growth and development in maternally HIVexposed and unexposed infants in Zambia. Clin Infect Dis. 2012; 54:434-442. [PubMed: 22247303]

7. Kovacs A, Schluchter M, Easley K, Demmler G, Shearer W, Russa PL, et al. Cytomegalovirus infection and HIV-1 disease progression in infants born to HIV-1-infected women. Pediatric Pulmonary and Cardiovascular Complications of Vertically Transmitted HIV Infection Study Group. N Engl J Med. 1999; 341:77-84. [PubMed: 10395631]

8. Chandwani S, Kaul A, Bebenroth D, Kim M, John D, Fidelia A, et al. Cytomegalovirus infection in human immunodeficiency virus type 1-infected children. Pediatric Infect Dis J. 1996; 15:310-314.

9. Nigro G, Krzysztofiak A, Gattinara GC, Mango T, Mazzocco M, Porcaro AM, et al. Rapid progression of HIV disease in children with cytomegalovirus DNAemia. AIDS. 1996; 10:11271133. [PubMed: 8874630]

10. Doyle M, Atkins JT, Rivera-Matos IR. Congenital cytomegalovirus infection in infants infected with human immunodeficiency virus type 1. Pediatric Infect Dis J. 1996; 15:1102-1106.

11. Griffiths PD. CMV as a cofactor enhancing progression of AIDS. J Clin Virol. 2006; 35:489-492. [PubMed: 16413825]

12. Bates M, Monze M, Bima H, Kapambwe M, Kasolo FC, Gompels UA. CIGNIS study group. High human cytomegalovirus loads and diverse linked variable genotypes in both HIV-1 infected and exposed, but uninfected, children in Africa. Virology. 2008; 382:28-36. [PubMed: 18929378]

13. Slogrove AL, Cotton MF, Esser MM. Severe infections in HIV-exposed uninfected infants: clinical evidence of immunodeficiency. J Trop Pediatr. 2010; 56:75-81. [PubMed: 19602487]

14. Filteau S. The HIV-exposed, uninfected African child. Trop Med Int Health. 2009; 14:276-287. [PubMed: 19171011]

15. Guibert G, Warszawski J, Le Chenadec J, Blanche S, Benmebarek Y, Mandelbrot L, et al. Decreased risk of congenital cytomegalovirus infection in children born to HIV-1-infected mothers in the era of highly active antiretroviral therapy. Clin Infect Dis. 2009; 48:1516-1525. [PubMed: 19388872]

16. Frederick T, Homans J, Spencer L, Kramer F, Stek A, Operskalski E, Kovacs A. The effect of prenatal highly active antiretroviral therapy on the transmission of congenital and perinatal/early postnatal cytomegalovirus among HIV-infected and HIV-exposed infants. Clin Infect Dis. 2012; 55:877-884. [PubMed: 22675157]

17. Chasela CS, Hudgens MG, Jamieson DJ, Kayira D, Hosseinipour MC, Kourtis AP, et al. Maternal or infant antiretroviral drugs to reduce HIV-1 transmission. N Engl J Med. 2010; 362:2271-2281. [PubMed: 20554982]

18. Boppana SB, Ross SA, Novak Z, Shimamura M, Tolan RW, Palmer AL, et al. Dried blood spot real-time polymerase chain reaction assays to screen newborns for congenital cytomegalovirus infection. JAMA. 2010; 303:1375-1382. [PubMed: 20388893]

19. Slyker JA, Lohman-Payne BL, John-Stewart GC, Maleche-Obimboc E, Emery S, Richardson B, et al. Acute cytomegalovirus infection in Kenyan HIV-infected infants. AIDS. 2009; 23:2173-2181. [PubMed: 19617812]

20. Mwaanza N, Chilukutu L, Tembo J, Kabwe M, Musonda K, Kapasa M, et al. High rates of congenital cytomegalovirus infection linked with maternal HIV infection among neonatal admissions at a large referral center in sub-Saharan Africa. Clin Infect Dis. 2014; 58:728-735. [PubMed: 24265360]

21. Khamduang W, Jourdain G, Sirirungsi W, Layangool P, Kanjanavanit S, Krittigamas P, et al. The interrelated transmission of HIV-1 and cytomegalovirus during gestation and delivery in the offspring of HIV-infected mothers. J Acquir Immune Defic Syndr. 2011; 58:188-192. [PubMed: 21792064]

22. Slyker J, Farquhar C, Atkinson C, Ásbjörnsdóttir K, Roxby A, Drake A, et al. Compartmentalized cytomegalovirus replication and transmission in the setting of maternal HIV-1 infection. Clin Infect Dis. 2014; 58:564-572. [PubMed: 24192386] 
23. Skolnik PR, Kosloff BR, Hirsch MS. Bidirectional interactions between human immunodeficiency virus type 1 and cytomegalovirus. J Infect Dis. 1988; 157:508-514. [PubMed: 2830343] 


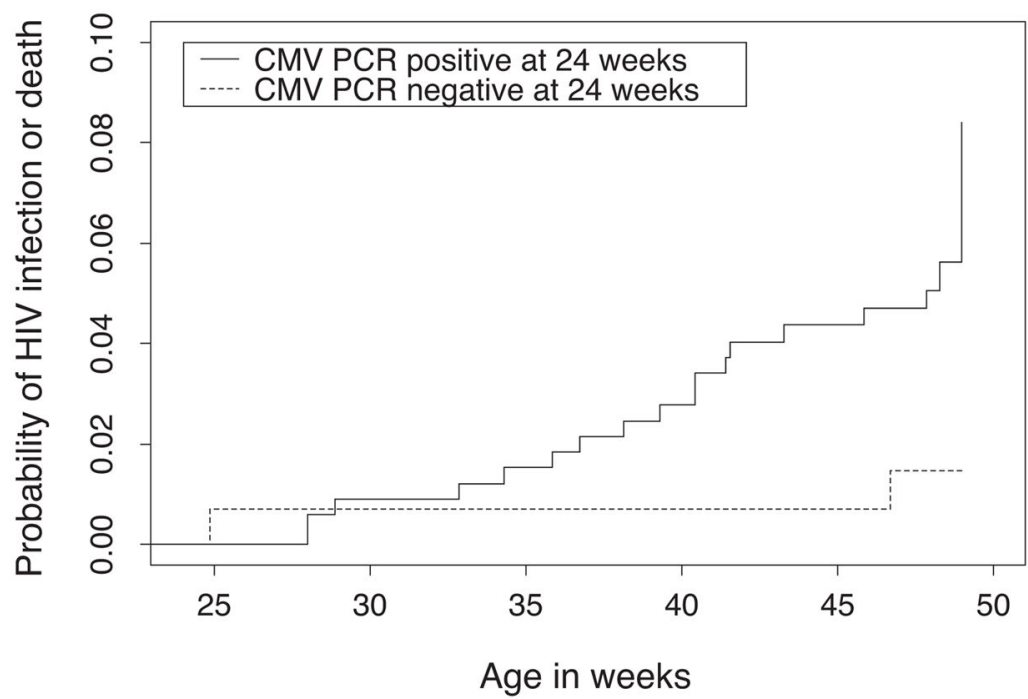

Fig. 1.

Kaplan-Meier estimates of the cumulative risk of infant HIV infection or death between 24 and 48 weeks of age by cytomegalovirus PCR result at 24 weeks. 


\section{Table 1}

Associations between infant cytomegalovirus PCR results at 24 weeks of age and infant morbidity/mortality and growth outcomes during 48 weeks of follow-up [The Breastfeeding, Antiretrovirals and Nutrition (BAN) Study, Lilongwe, Malawi].

\begin{tabular}{lcc}
\hline & CMV PCR at 24 weeks \\
\cline { 2 - 3 } & Hazard ratio (95\% CI) & P value \\
\hline HIV infection (2-48 weeks) & $1.99(0.57-6.92)$ & 0.28 \\
HIV infection (24-48 weeks) & $4.52(0.58-35.3)$ & 0.15 \\
Infant death & $4.05(0.51-32.0)$ & 0.18 \\
HIV infection or infant death (2-48 weeks) & $2.49(0.86-7.21)$ & 0.09 \\
HIV infection or infant death (24-48 weeks) & $4.27(0.99-18.4)$ & 0.05 \\
Severe pneumonia/SFI & $1.31(0.78-2.18)$ & 0.31 \\
Severe diarrhea or growth faltering & $0.79(0.38-1.66)$ & 0.54 \\
Severe malaria & $1.51(0.47-4.84)$ & 0.49 \\
\hline & Mean difference in Z-score (95\% CI) & $P$ \\
& & $P$ value \\
\hline Weight-for-age & $0.02(-0.17-0.22)$ & 0.81 \\
Length-for-age & $0.11(-0.08-0.29)$ & 0.27 \\
\hline
\end{tabular}

CI, confidence interval; CMV, cytomegalovirus; SFI, serious febrile illness.

${ }^{a}$ All hazard ratios and coefficients adjusted for antiretroviral treatment group. 